Appl. Phys. B 49, 331-337 (1989)

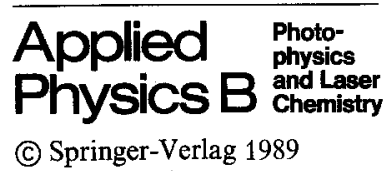

\title{
Compact, Wide Aperture X-Ray Preionized XeCl Laser with High Specific Optical Power
}

\author{
M. Steyer, K. A. Stankov ${ }^{\star}$, H. Mizoguchi ${ }^{\star \star}$, B. Ouyang $\star \star \star$, and F. P. Schäfer \\ Max-Planck-Institut für biophysikalische Chemie, Abteilung Laserphysik, \\ Postfach 28 41, D-3400 Göttingen, Fed. Rep. Germany
}

Received 20 February 1989/Accepted 16 May 1989

\begin{abstract}
An X-ray preionized, discharge-pumped XeCl laser with a variable beam crosssection of up to $6 \times 6 \mathrm{~cm}^{2}$ is described. It uses flat electrodes and the beam width is determined by $\mathrm{X}$-ray collimation. Its operation characteristics concerning reduced electric field strength $(E / p)$ and X-ray dose are discussed in detail. The inductance of the discharge loop is minimized using a water capacitor arrangement. A very high specific optical power $(90 \mathrm{MW} / 1)$ is achieved in an active volume of 1.21 . The pulse energy exceeds $5 \mathrm{~J}$ in a $45 \mathrm{~ns}$ pulse (FWHM).
\end{abstract}

PACS: $42.55 \mathrm{G}, 42.60 \mathrm{~B}, 52.80 \mathrm{H}$

For the generation of intense UV laser pulses at the $\mathrm{KrF}$ and $\mathrm{XeCl}$ wavelengths, a wide aperture, discharge-pumped excimer laser with a high excitation rate is of considerable interest. However, when scaling discharge-pumped systems to a larger cross-section (active volume) one faces the problem that the energy storage medium has to be low-inductively coupled to the main discharge. For pumping large volumes a pulse forming line (PFL) is usually employed and connected by a rail gap switch to the laser head to decrease the voltage rise-time [1]. However, the pumping rate is limited by the given impedance of the PFL. In contrast to the total pump energy, the pump power is not increased with the length of the line. The achievable optical power per unit volume is generally considerably smaller than in small volume systems which mostly employ lumped circuits. It seldom exceeds $50 \mathrm{MW} / 1$ for active volumes of more than 11 , whereas in small lasers $\left(\approx 50 \mathrm{~cm}^{3}\right), 300 \mathrm{MW} / \mathrm{L}$ can be easily obtained [2].

To achieve high pumping rates in a large aperture laser employing a lumped circuit, both low inductance

Permanent addresses: * Department of Physics, University of Sofia, BG-1126 Sofia, Bulgaria

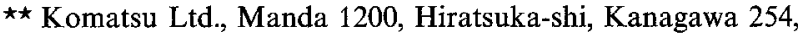
Japan

$\star \star \star$ Institute of Optics and Fine Mechanics, Department of Plasma Physics, Academia Sinica, Shanghai, P.R. China electrical circuit and high charging voltage are needed. We employ a combination of the waterline and the lumped circuit technology by using a number of parallel waterlines of short electrical length [3]. This arrangement has a very low inductance and is suitable for a charging voltage of $150 \mathrm{kV}$.

$\mathrm{X}$-ray preionization was chosen to provide a sufficiently high and spatially homogeneous initial electron distribution even at high pressures. Moreover, $\mathrm{X}$-ray preionization allows easy variation of the beam cross-section and the use of flat electrodes $[4,5]$. By variation of the beam width which is determined by horizontal lead apertures, the pump current density can be varied and optimized.

In this paper we report on a wide aperture $\left(6 \times 6 \mathrm{~cm}^{2}\right) \mathrm{XeCl}$ oscillator. The active volume of the laser can be varied up to 1.51 . Under optimized conditions more than $5 \mathrm{~J}$ are generated in a $45 \mathrm{~ns}$ (FWHM) pulse corresponding to $4 \mathrm{~J} / 1$ and an average specific optical power of $90 \mathrm{MW} / \mathrm{l}$. We performed detailed studies of the influence of electrical parameters and conditions of X-ray preionization.

\section{Experimental}

For the low-inductance discharge circuit we have modified the water-capacitor design of Cirkel et al. [3]. 


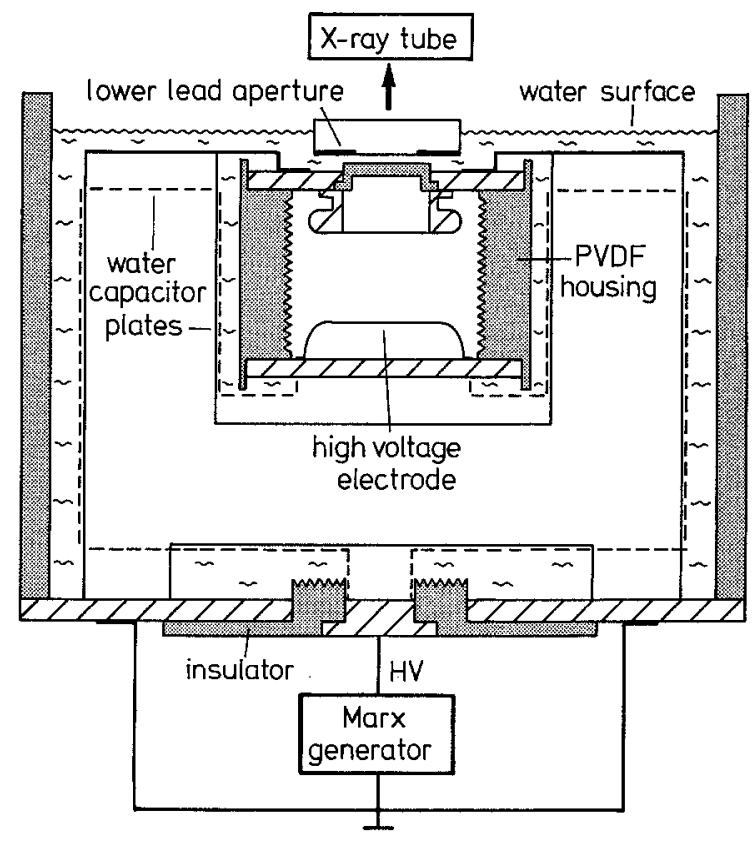

Fig. 1. Cross-section of the excimer laser system showing the laser head and the water capacitor arrangement

Two types of stainless steel plates, each differently shaped, are alternately connected to the laser electrodes and inserted into a tank filled with deionized water. Figure 1 shows this arrangement viewed along the optical axis. Two adjacent plates have a separation of $14 \mathrm{~mm}$. The use of water has the well-known advantages for high-voltage applications. Its high dielectric constant allows large energy storage density and a relatively compact design. Water capacitors can be exposed to very high voltage. Voltage and current reversal do not affect the capacitor lifetime and occasionally occurring electrical breakdowns do not cause irreversible damage. Due to the large number (9-15) of parallel capacitors, their contribution to the discharge loop inductance is very small [3]. It is estimated to be more than one order of magnitude smaller than the geometrical laser head inductance $(\approx 15 \mathrm{nH})$.

The water capacitor arrangement can be visualized as a number of parallel waterlines with a double electrical transit time of about $15 \mathrm{~ns}$. However, since the double transit time is short compared to typical times in the discharge loop (e.g., the current rise-time), the water capacitor cannot act as a pulse forming line, and a lumped-circuit behaviour is expected.

The laser head is made from polyvinylidenfluoride (PVDF). The upper electrode is made of aluminum, coated with a $300 \mu \mathrm{m}$ layer of nickel. It has a $6 \mathrm{~cm}$ wide slot milled along its length to allow maximum X-ray penetration through the remaining $1 \mathrm{~mm} \mathrm{Al}$ and the $1 \mathrm{~cm}$ thick PVDF cap. The lower electrode consists of a
$1 \mathrm{~mm}$ thick stainless steel plate bent to shape. Both electrodes are flat in a central region of more than $8 \mathrm{~cm}$ width. Their length is about $40 \mathrm{~cm}$ and their separation $d$ can be varied by the insertion of spacers. In the experiments reported here $d$ was kept constant at $6 \mathrm{~cm}$.

The water capacitor $C_{\mathrm{P}}(60 \mathrm{nF}$ or $100 \mathrm{nF})$ formed by the stacked stainless steel plates with the water as dielectric is charged by a $n$-stage $(n=3-4)$ Marx generator which is situated below the water tank. Each stage of the Marx generator has a capacitance of $C_{0}=330 \mathrm{nF}$ and can be charged up to $U_{0}=40 \mathrm{kV}$. The erected Marx generator, the charging loop inductance $(\approx 480 \mathrm{nH})$ and the water capacitor form a charge transfer circuit. When no breakdown occurs in the laser channel, the voltage on $C_{\mathrm{P}}$ rises up to its first peak value, $U_{\mathrm{P}}$, typically within $\delta t \approx 250 \mathrm{~ns}(10-90 \%)$, both $U_{\mathrm{P}}$ and $\delta t$ depending on the number of Marx stages and $C_{\mathrm{p}}$. In the following $U_{\mathrm{p}}$ is denoted as the peak charging voltage of the water capacitor.

The X-ray diode consists of a cold graphite cathode and a $15 \mu \mathrm{m}$ thick tantalum foil anode in transmission geometry. It is driven by a four-stage Marx generator, each stage $(38 \mathrm{nF})$ being charged to $30 \mathrm{kV}$. A peaking water capacitor of $4 \mathrm{nF}$ further increases the voltage across the tube [5]. The X-ray tube provides a pulse of 100 ns length (FWHM) and a dose of nearly $200 \mathrm{mR}$ (measured with a pen dosimeter) in front of the anode. It decreases to approximately $15 \mathrm{mR}$ inside the laser chamber due to beam divergence and attenuation. Severe attenuation of the soft spectral components $(\leqq 30 \mathrm{keV})$ is caused predominantly by the nickel coating of the electrode. The anode of the $\mathrm{X}$-ray tube is placed $25 \mathrm{~cm}$ above the lower electrode surface. The radiation is collimated by two horizontal lead apertures. One aperture of fixed width $(3.0 \mathrm{~cm})$ is placed directly below the $\mathrm{X}$-ray anode and the other aperture of variable width is placed as close as possible to the upper laser electrode.

The optical windows of the laser head consist of $20 \mathrm{~mm}$ thick fused quartz plates (Suprasil). A quartz plate covered with a $100 \%$ reflective coating is used as the back mirror while the output window remains uncoated.

The excimer laser operates at one shot every ten seconds.

\section{Results and Discussion}

\subsection{Dependence of Laser Characteristics on $E / p$}

The laser was operated with an optimized (standard) $\mathrm{XeCl}$ gas mixture consisting of $1.33 \% \mathrm{Xe}, 0.07 \% \mathrm{HCl}$ and $\mathrm{Ne}$ as the buffer gas. Particularly in X-ray preionized lasers, $\mathrm{Ne}$ is superior compared to $\mathrm{He}$ because of higher X-ray absorption [2]. In order to 


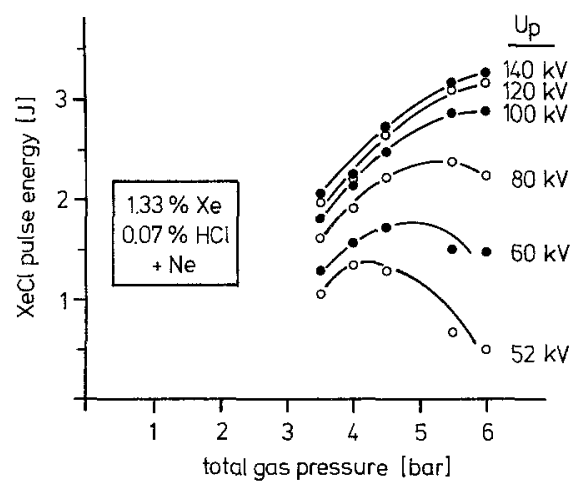

Fig. 2. Laser pulse energy $E_{\mathrm{XeCl}}$ as a function of total gas pressure with the peak capacitor voltage $U_{p}$ as parameter

keep the kinetic constants of the laser gas and the preionization electron density easily comparable during the course of experiments, the relative concentrations of the active gases were kept constant.

In Fig. 2 a typical variation of the optical pulse energy $E_{\mathrm{XcCl}}$ as a function of the gas pressure $p$ is shown with $U_{\mathrm{P}}$ as parameter. These results have been obtained with a four-stage Marx generator and $C_{\mathrm{P}}=60 \mathrm{nF}$. Here $U_{0}=10 \mathrm{kV}$ charging of the Marx generator corresponds to $40 \mathrm{kV}$ peak voltage $\left(U_{\mathrm{P}}\right)$ on the water capacitors. Hence, the energy transfer efficiency is about 0.73 . However, gas breakdown occurs before $U_{\mathrm{P}}$ is reached. The breakdown voltage was not recorded systematically as a function of charging voltage and gas pressure. Due to the slow voltage rise across the laser electrodes, the breakdown voltage is not very much higher $(\leqq 20 \%)$ than $U_{\mathrm{S}}$, the static selfbreakdown voltage of the gas mixture [6]. Depending on the total pressure, the laser pulse duration (FWHM) varies between $\approx 35 \mathrm{~ns}$ ( $p=6$ bar) and $\approx 60 \mathrm{~ns}$ ( 3 bar). A significant variation of the pulse duration with the charging voltage was not observed.

The laser was operated at pressures up to 6 bar and voltages up to $U_{\mathrm{P}}=140 \mathrm{kV}$. Figure 2 demonstrates two particular features. Firstly, over the whole investigated voltage range an increase of voltage results in an increase of pulse energy. Secondly, when the gas pressure $p$ is varied at constant voltage, an energy maximum is observed at low voltages. At higher voltages this maximum is obviously outside the investigated pressure range.

According to Fig. 2, there is an optimum value for $(E / p)$, which results in a maximum laser energy when the pressure is changed. For higher pressure, higher voltage is needed to reach this optimum. Let us hence refer each voltage to the pressure and denote by $(E / p)_{\mathrm{p}}$ and $(E / p)_{\mathrm{S}}$ the reduced field strengths, obtained by dividing $U_{\mathrm{P}}$ and $U_{\mathrm{S}}$, respectively, by the gas pressure $p$ and the electrode distance $d$. The variations of Fig. 2 appear to be determined by the reduced electric field strength $(E / p)_{\mathbf{p}}$. This is well illustrated by the dependence of the intrinsic efficiency $\eta\left(:=E_{\mathrm{XeCl}} / \frac{1}{2} C_{p} U_{p}^{2}\right)$ on $(E / p)_{\text {p }}$, which is depicted in Fig. 3 for the same data used in Fig. 2. Measured for various combinations of voltages and pressures, the data points fit reasonably well to a common curve. Below a threshold value of about $1.0 \mathrm{kV} /(\mathrm{cm} \cdot \mathrm{bar})$ no laser action is observed. This threshold value is, within the experimental uncertainty, equal to the self-breakdown field strength

$(E / p)_{s} \approx 1.1 \mathrm{kV} /(\mathrm{cm} \cdot \mathrm{bar})$

calculated from the data given by Taylor [6]. At slightly higher field strengths than $(E / p)_{\mathrm{S}}\left[(E / p)_{\mathrm{P}}\right.$ $\approx 1.5 \mathrm{kV} /(\mathrm{cm} \cdot \mathrm{bar})]$ a highest intrinsic efficiency of more than $3 \%$ is obtained. At still higher field strengths $\eta$ gradually decreases, although the total laser energy continues to grow.

This behaviour of the efficiency has been observed earlier in a XeCl laser employing a long pulse forming line (PFL) [7]. Those data were in quantitative agreement with the following equation $[7,8]$ :

$$
\begin{aligned}
\eta \propto \eta_{\mathrm{ET}} & =1-\left(\frac{2 \cdot U_{\mathrm{D}}-U_{\mathrm{C}}}{U_{\mathrm{C}}}\right)^{2} \\
& =\frac{4 \cdot(E / p)_{\mathrm{D}} \cdot\left[(E / p)_{\mathrm{C}}-(E / p)_{\mathrm{D}}\right]}{(E / p)_{\mathrm{C}}^{2}} .
\end{aligned}
$$

$\eta_{\mathrm{ET}}$ is the energy transfer efficiency from the PFL to the discharge. $U_{\mathrm{D}}$ is the steady-state (sustaining) voltage across the discharge and $U_{\mathrm{C}}$ is the line charging voltage. Since a constant electrical pulse length has to be assumed, (1) is not applicable in the present lumped circuit. However, there is a qualitative similarity between our efficiency data and that in [7]. In both cases, the laser efficiency (Fig. 3) has already dropped to zero at $(E / p)_{\mathrm{S}}$ and not, as may be concluded from (1), at $(E / p)_{\mathrm{D}}$, since the sustaining electrical field strength is

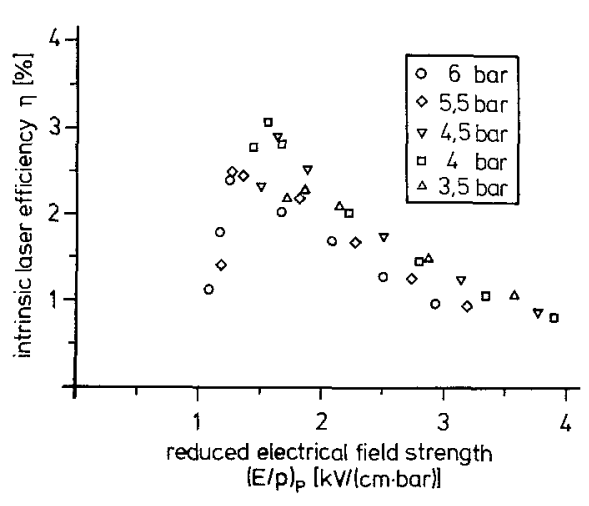

Fig. 3. Dependence of the intrinsic laser efficiency $\eta$ $\left(:=E_{\mathrm{XeCl}} / \frac{1}{2} C_{\mathrm{P}} U_{\mathrm{P}}^{2}\right)$ on the reduced electric field strength $(E / p)_{\mathrm{P}}$, measured at various pressures and voltages. If the efficiency is related to the energy stored in the Marx generator $\left(=\frac{1}{2} n C_{0} U_{0}^{2}\right), \eta$ must be multiplied by a constant factor of 0.73 


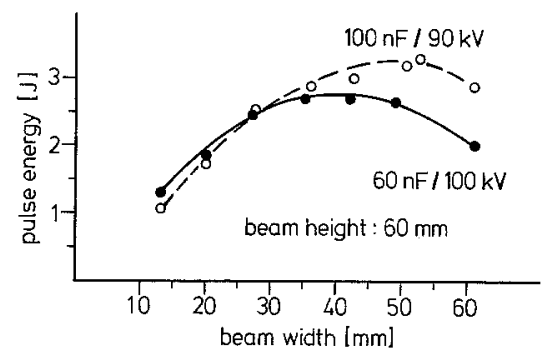

Fig. 4. Dependence of laser energy on beam width for two different capacitor sizes ( $p=6$ bar)

considerably smaller than the self-breakdown field strength. For an exact evaluation of $(E / p)_{\mathrm{D}}$, a number of gas kinetic processes have to be taken into account $[9,10]$. Generally, $(E / p)_{\mathrm{D}}$ is of the order of $0.5 \mathrm{kV} /(\mathrm{cm} \cdot$ bar $)[3,7]$.

A homogeneous glow discharge acts as a constantvoltage load, so that the sustaining voltage $U_{\mathbf{D}}$ is a function of the gas composition only and not of time or discharge current $I[1,8]$. This also applies for lumped circuits. However, here the discharge current during the "stable" phase may change with time and, therefore, the measurable voltage across the discharge, $U=U_{\mathrm{D}}+L d I / d t$ may not show a plateau $[3,10]$. Since the sustaining field strength across the active gas is only a function of the gas composition, an increase of pump power can only be achieved by a higher current provided by the electrical network.

By changing the width of the lower X-ray aperture the width of the laser beam can be varied [5]. Since the discharge stabilizes at a certain voltage $U_{\mathrm{D}}$ (which should be independent of the beam width) and the total current is constant (since it is mainly determined by the size of the water capacitor, the breakdown voltage and the discharge loop inductance), the current density will be approximately inversely proportional to the beam width $w$. The laser pulse energy as a function of the beam width $w$ is shown in Fig. 4 for two different values of $C_{\mathrm{p}}$. Displayed is the total pulse energy as calculated from the measured value (GenTec ED 500, sensitive area $4.5 \times 4.5 \mathrm{~cm}^{2}$ ) and the beam cross-section, as observed with UV-sensitive paper. The pulse duration does not significant change with $w$. For narrow apertures a linear growth of the pulse energy with $w$ is observed indicating a constant optical energy density. However, for larger widths the output energy maximizes and then decreases. If the beam becomes wider than $6 \mathrm{~cm}$, the discharge additionally becomes inhomogeneous due to the enhanced electrical field strength at the electrode edges.

The optimum beam width changes to a higher value if the capacitor $C_{\mathrm{P}}$ is larger (Fig. 4). A larger capacitance provides a larger current, which corre- sponds (at a given optimum current density) to a larger beam width.

The highest pulse energy (5.1 J) achieved so far has been obtained with a three-stage Marx generator, $U_{\mathrm{P}}=136 \mathrm{kV}, C_{\mathrm{P}}=60 \mathrm{nF}$, the standard gas mixture (total pressure 6 bar) containing $0.02 \% \mathrm{H}_{2}$ and a beam width of $4.7 \mathrm{~cm}$. The pulse length was $45 \mathrm{~ns}$ (FWHM). This result was obtained using a fresh gas mixture while for the systematic studies reported in this paper we have used older gas mixtures in which the pulse energy is typically $30 \%$ smaller.

\section{2. $X$-Ray Preionization}

Preionization of high pressure discharge pumped gas laser represents an essential part of the whole system. If the initially present electron density at voltage breakdown is too small or not sufficiently homogeneous, the occurrence of arcs will disturb the laser performance and the highest possible output is not obtained.

The use of X-ray preionization allows a relatively easy quantification of the preionization strength in terms of X-ray dose and its variation by the insertion of $\mathrm{X}$-ray absorbing materials. The measurement of the transient electron density is not a trivial task [11], since it is strongly influenced by dissociative attachment of electrons to halogen molecules [6]. Additionally, the evaluation of the electron density is difficult because the $\mathrm{X}$-ray spectrum is not known in detail and a multitude of atomic processes (fluorescence radiation, Auger electrons) as well as geometrical factors, laser voltage rise-time, X-ray pulse shape etc. have to be considered [11].

Therefore, we will state only the dose and the pulse length (which does not vary with attenuation) and will assume that with a given gas mixture the preionization electron density $n_{\mathrm{e}}$ is proportional to the dose $D$. We will also assume that $n_{\mathrm{e}}$ follows linearly the total gas pressure $p$ when the relative concentrations of the active gases are kept constant. We believe this is a reasonable approximation, since the photoelectric absorption constants $\mu\left[\mathrm{cm}^{-1}\right]$ of the active gases have a practically constant ratio independent of the quantum energy above $34.6 \mathrm{keV}$ (Xe $K$-edge). Photons of lower energy can practically not penetrate the nickel coating of the upper electrode.

In the literature a logarithmic growth of the laser pulse energy, $E_{\mathrm{L}}$, with the preionization electron density $n_{e}$ has often been observed, which has (at least partially) been attributed to an increase of optical pulse length [12-14]. This dependence can be expressed as

$E_{\mathrm{L}}=c \ln \left(n_{\mathrm{e}} / n_{\mathrm{e}, \min }\right)$,

where $c$ is a constant and $n_{\mathrm{e}, \min }$ is the minimum electron density, below which no lasing can be ob- 
served. The limitation of (2) is obvious, since it does not describe the energy saturation which is observed at high electron densities $[2,6]$. To take into account this saturation, we propose, as an empirical generalization of (2), the model function

$E_{\mathrm{L}}=E_{\text {sat }}\left\{1-\exp \left[-\alpha \cdot\left(n_{\mathrm{e}}-n_{\mathrm{e}, \min }\right)\right]\right\}$.

Since $n_{\mathrm{e}}$ and the X-ray dose $D$ are assumed to be proportional, $n_{\mathrm{e}}$ can be replaced by $D$ in this formula giving

$E_{\mathrm{L}}=E_{\text {sat }}\left\{1-\exp \left[-\alpha^{\prime} \cdot\left(D-D_{\min }\right)\right]\right\}$.

For small enough $n_{\mathrm{e}}$, (3) describes a logarithmic growth as in (2). For large $n_{\mathrm{e}}, E_{\mathbf{L}}$ converges to the saturation energy $E_{\text {sat }}$.

We also used an alternative model function, namely

$E_{\mathrm{L}}=E_{\mathrm{sat}}\left\{1-\exp \left[-\alpha^{\prime \prime} \cdot \ln \left(D / D_{\min }\right)\right]\right\}$.

Although this function becomes equivalent to (2) for small $D$, it generally does not provide such good agreement with the experimental data as (3) for larger D.

Several models have been developed to derive the minimum electron density necessary for the formation of a homogeneous glow discharge $[15,16]$. It is generally agreed that threshold is reached when spatial overlap occurs between the avalanches of the initial electrons. Otherwise, arcs are formed during the formation phase of the discharge, and the gas is not effectively pumped. However, the avoidance of streamers alone is not sufficient for optimum pumping conditions, since the avalanche formation time decreases with higher initial electron density which results in a longer optical pulse [12-14]. Additionally, when the number density of preionization electrons is too small, the optimum electron density in a fully established discharge $\left(\approx 10^{15} \mathrm{~cm}^{-3}\right)$ may not be reached at all.

At an insufficient electron density, both pulse shortening and decrease of laser intensity are usually observed in our experiments. Contrary to [6], we denote the smallest dose $D_{\min }$ (the electron density) necessary for any laser output as the threshold value at which - somewhat arbitrarily $-90 \%$ of the possible pulse energy is reached, as the saturation value $D_{\text {sat }}$ $\left(n_{\mathrm{e}, \mathrm{sat}}\right)$.

In Fig. 5 the dependence of the laser pulse energy on the preionization dose is shown for the standard gas mixture for various total pressures. To allow a comparison with Fig. 2, $U_{\mathrm{P}}$ has been kept constant at $120 \mathrm{kV}$. A relative $\mathrm{X}$-ray dose of 1 corresponds to $15 \mathrm{mR}$ inside the chamber. The X-ray pulse length is $100 \mathrm{~ns}$ (FWHM). For all different pressures the well known linear growth of $E_{\mathrm{XeCl}}$ in a logarithmic plot and

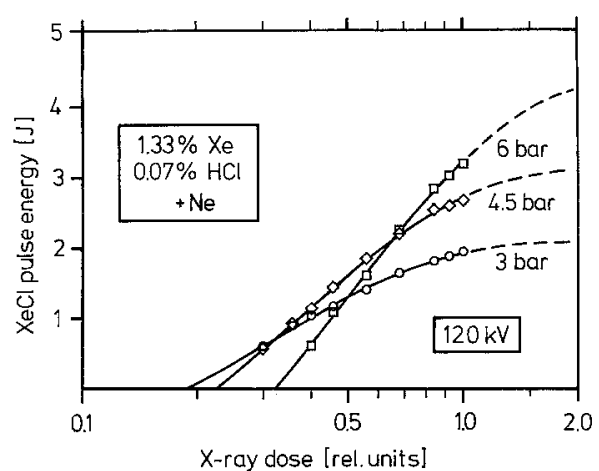

Fig. 5. Variation of the laser pulse energy with $X$-ray dose, for various pressures, when the charging voltage is kept constant $\left(U_{\mathrm{p}}=120 \mathrm{kV}\right) ; 1$ relative unit corresponds to $15 \mathrm{mR}$ in $100 \mathrm{~ns}$ (FWHM) inside the laser chamber

some kind of saturation is observed. When comparing the three curves, a number of features are evident.

Figure 5 shows clearly that $D_{\min }, \alpha^{\prime}$ (the slope), and $E_{\text {sat }}$ become larger with higher pressure. At the highest available X-ray dose (1 relative unit) and a peak capacitor voltage of $120 \mathrm{kV}$ the output energy increases in the order 3 bar, $4.5 \mathrm{bar}, 6 \mathrm{bar}$, as has been discussed earlier. However, at lower doses this order is changed in various ways. It is thus apparent that the pressure dependence of the laser pulse energy is strongly dependent on the preionization parameters.

For a quantitative analysis of the experimental data, the model function (3) was fitted to the points yielding the threshold dose $D_{\min }$, the slope $\alpha^{\prime}$ and the saturation energy $E_{\text {sat }}$. Whereas for different model functions $D_{\min }$ and $\alpha^{\prime}$ are practically uniquely determined, $E_{\text {sat }}$ is not always and has therefore to be treated with care.

The threshold value $D_{\text {min }}$ grows as expected with the total gas pressure. Below a certain dose $(\approx 0.3$ relative units) the pulse energy could not be measured with satisfactory precision since the shot-to-shot fluctuations were large. Therefore, the energy dependence for small doses is not exactly known. However, we assume a linear dependence (3), because we did not observe any significant deviation from it.

In Fig. 6 the minimum doses $D_{\min }$ derived in this way are plotted as a function of pressure. Only slightly dependent on the model function used, they follow reasonably well a power law $D_{\text {min }} \propto p^{x}$, with $x=0.9 \pm 0.2$. If we further assume that with a given dose the electron density scales with the total pressure, we find $n_{\mathrm{e}, \min } \propto p^{y}$, with $y=1.9 \pm 0.2$. The same pressure dependence holds for the saturation dose, which is, by definition, given by

$D_{\text {sat }}=(\ln 10) / \alpha^{\prime}+D_{\min }$,

as is also depicted in Fig. 6. We therefore conclude that both the threshold electron density $n_{\mathrm{e}, \min }$ and the 


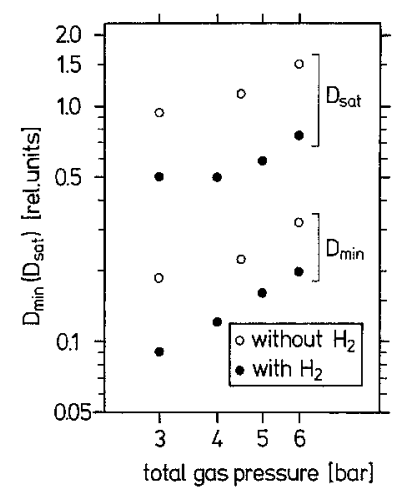

Fig. 6. Dependence of the threshold dose $D_{\min }$ and the saturation dose $D_{\text {sat }}$ on gas pressure $\left(U_{\mathrm{P}}=\right.$ const $)$

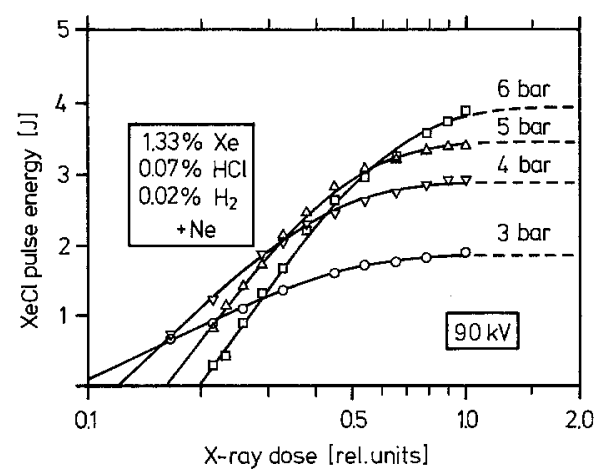

Fig. 7. Same as Fig. 5, but for a gas mixture containing $0.02 \% \mathrm{H}_{2}$ $\left(U_{\mathbf{P}}=90 \mathrm{kV}\right)$

saturation electron density $n_{\mathrm{e}, \text { sat }}$ scale approximately with the square of the total pressure $p$ when $U_{\mathrm{P}}$ is kept constant.

In Fig. 7 the same experiment is carried out as in Fig. 5, but with a gas mixture containing a small amount $(0.02 \%)$ of $\mathrm{H}_{2}$. This gas is frequently used in $\mathrm{XeCl}$ lasers since it increases the gas lifetime by replenishing the $\mathrm{HCl}$ concentration [17]. Under identical experimental conditions we typically observed a higher pulse energy with $\mathrm{H}_{2}$, and also the sensitivity of laser output to a reduction of X-ray dose was much smaller. Both the threshold and the saturation dose are considerably smaller (Fig. 6). However, the saturation energy seems to be higher without $\mathrm{H}_{2}$. The pressure dependence of $D_{\min }$ and $D_{\text {sat }}$ follows practically the same approximate square power law. Additionally, an increase of the preionization electron lifetime is observed by a larger time delay being allowed between $\mathrm{X}$-ray preionization and main discharge. A reason for this may be that $\mathrm{H}_{2}$ inhibits the formation of $\mathrm{Cl}_{2}$ which is one of the most important loss channels for preionization electrons [13].

It is important to note that the curves of Fig. 5 and Fig. 7 have been measured with a constant charging voltage $U_{\mathrm{P}}$. Therefore, the value for $E / p$ at gas break- down, which is responsible for electron acceleration, is changed. Additionally, the effective rise-time $\delta t$ of the laser voltage (until breakdown) decreases with smaller pressure. Both faster rise-time [13] and higher $E / p$ [14] are expected to reduce the requirement on the preionization electron density.

This was observed by keeping $p$ constant and changing the charging voltage $U_{\mathrm{p}}$. If $U_{\mathrm{p}}$ is increased at constant $p$, the effective voltage rise-time becomes smaller, and the minimum dose $D_{\min }$ is reduced. This dependence follows approximately a $D_{\min } \propto U_{\mathrm{P}}^{-z}$ law, with $z=0.4 \pm 0.2$.

Consequently, if $U_{\mathrm{P}}$ is kept constant, a combined effect of the varying pressure and the varying voltage rise-time is observed. To keep $\delta t$ constant, $U_{\mathrm{P}}$ was changed with the pressure so that $(E / p)_{\mathrm{P}}$ remains constant. The exponent $y$ of the power law $n_{\mathrm{e}, \min } \propto p^{y}$ becomes $y=1.6 \pm 0.2$. The plots of the functional dependence of $E_{\mathrm{XeCl}}$ on $D$ for various pressures intersect, just as for constant $U_{\mathrm{P}}$ (Figs. 5, 7). This is a direct consequence of both $D_{\min }$ and $E_{\text {sat }}$ growing with $p$.

The dependence of the minimum electron density for homogeneous glow discharge had been derived for an instantaneously switched on electric field [16] $\left(\propto p^{3}\right.$, see also [18] and references therein) and taking into account a finite voltage rise-time on the electrodes $\left(\propto p^{3 / 2}\right)$ [15], both laws being applicable for constant $(E / p)$. Considering the relatively long voltage rise-time in the present laser, the agreement of the observed dependence with the theory of Levatter [15] is quite satisfactory.

Compared to previous results [2] a considerably higher (approximately 10 times) minimum X-ray dose is required to achieve laser output. The reason for this discrepancy is not fully understood, but a possible explanation could be the present flat electrode profile. The electric field strength is not constant along the electrode width due to its finite dimension. Therefore, the preionization electrons are differently accelerated, which, particularly at low initial electron densities and small electric field strengths, may lead to discharge inhomogeneities. Another reason may be the small value of $(E / p)$ at gas breakdown due to the slow voltage rise compared to the earlier reported value [2].

\section{Conclusion}

We have demonstrated a discharge pumped $\mathrm{XeCl}$ laser with a wide aperture $\left(\leqq 6 \times 6 \mathrm{~cm}^{2}\right)$ and a volume of about 11 using flat electrodes. Under optimized conditions, $5.1 \mathrm{~J}$ optical energy has been extracted in a $45 \mathrm{~ns}$ (FWHM) pulse. This corresponds to a specific optical power of $90 \mathrm{MW} / 1$ which is to our knowledge the highest value reported so far for a laser of this size. 
A low inductance water capacitor design has been employed. Due to slow voltage rise across the laser electrodes, gas breakdown occurs close to the static self-breakdown field-strength $(E / p)_{\mathrm{S}} \approx 1.1 \mathrm{kV} /$ $(\mathrm{cm} \cdot \mathrm{bar})$. The intrinsic efficiency of the laser is only a function of the reduced electrical field strength $(E / p)_{\mathrm{P}}$ given by the peak charging voltage. The laser pulse energy increases monotonically with the charging voltage. Highest efficiency is achieved for a slightly higher field strength $\left[(E / p)_{\mathrm{P}} \approx 1.5 \mathrm{kV} /(\mathrm{cm} \cdot \mathrm{bar})\right]$ than $(E / p)_{s}$.

We have studied the dependence of the laser output energy on the X-ray preionization strength. An empirical model function has been proposed to describe this dependence including saturation at high doses. The minimum electron density $n_{e, \min }$ necessary to achieve a homogeneous glow discharge and laser output varies with the total pressure $p$ of the gas according to $p^{y}$ with $y=1.9 \pm 0.2$, if the peak charging voltage $U_{\mathrm{p}}$ is kept constant, and as $p^{x}$ with $x=1.6 \pm 0.2$, if $(E / p)_{\mathrm{P}}$ is kept constant. With a small amount of $\mathrm{H}_{2}(0.02 \%)$ added to the gas mixture, the pulse energy is usually higher and the sensitivity to the $\mathrm{X}$-ray dose considerably lower. However, without $\mathrm{H}_{2}$ and a higher X-ray dose the output may probably be further increased. This may be done by increasing the number of stages of the Marx generator.

Acknowledgements. K. A. Stankov acknowledges a research fellowship from the Alexander-von-Humboldt Foundation. $\mathrm{H}$. Mizoguchi acknowledges a research scholarship from Komatsu Ltd. This work has been partly financed by the „Bundesministerium für Forschung und Technologie“ (BMFT) and by the „Deutsche Forschungsgemeinschaft" (DFG) through its Leibniz program. We would like to thank Dr. G. York and J. Jethwa for their contributions in the early stage of experimental set-up and Dr. B. Rácz for help during some of the measurements. We are indebted to J. Jethwa for a critical reading of the manuscript.

\section{References}

1. R.S. Taylor, P.B. Corkum, S. Watanabe, K.E. Leopold, A.J. Alcock: IEEE J. QE-19, 416 (1983)

2. M. Steyer, H. Voges: Appl. Phys. B 42, 155 (1987)

3. H.J. Cirkel, W. Bette, D. Friede, R. Müller: Proc. SPIE 735, 50 (1987)

H.-J. Cirkel, W. Bette: German Patent DE2932781 C2

4. C.R. Tallman, I.J. Bigio: Appl. Phys. Lett. 42, 149 (1983)

5. M. Steyer, B. Ouyang, K.A. Stankov, G. Szábo, H. Mizoguchi, F.P. Schäfer: Proc. SPIE 1023, 75 (1989)

6. R.S. Taylor: Appl. Phys. B 41, 1 (1986)

7. M.R. Osborne, P.W. Smith, M.H.R. Hutchinson: Opt. Commun. 52, 415 (1985)

8. D.E. Rothe, C. Wallace, T. Petach: AIP Conf. Proc. 100 (Excimer Lasers), 33 (1983)

9. H. Hokazono, K. Midorikawa, M. Obara, T. Fujioka: J. Appl. Phys. 56, 680 (1984)

10. G. Stielow, Th. Hammer, W. Bötticher: Appl. Phys. B 47, 333 (1988)

11. M.R. Osborne: J. Appl. Phys. 63, 32 (1988)

12. S. Sumida, K. Kunitomo, M. Kaburagi, M. Obara, T. Fujioka: J. Appl. Phys. 52, 2682 (1981)

13. H. Shields, A.J. Alcock, R.S. Taylor: Appl. Phys. B 31, 27 (1983)

14. D. Lo: Appl. Phys. B 48, 405 (1989)

15. J.I. Levatter, S.-C. Lin: J. Appl. Phys. 51, 210 (1980)

16. A.J. Palmer: Appl. Phys. Lett. 25, 138 (1974)

17. T.J. McKee, D.J. James, W.S. Nip, R.W. Weeks, C. Willis: Appl. Phys. Lett. 36, 943 (1980)

18. A. Atanasov, S.G. Vasilev, I.O. Kovalyov, G.P. Kuz'min, A.A. Nesternko: J. Phys. D 21, 1750 (1988) 